Arq. Bras. Med. Vet. Zootec., v.56, n.6, p.741-744, 2004

\title{
Ocorrência de Babesia sp em pequenos roedores no Brasil
}

\author{
[Occurrence of Babesia sp in small rodents in Brazil] \\ G.S. Gazeta ${ }^{1}$, R.W. Carvalho ${ }^{1,3}$, R.F. Avelar ${ }^{2}$, M. Amorim ${ }^{1}$, A.E. Aboud-Dutra ${ }^{1}$ \\ ${ }^{1}$ Departamento de Entomologia, Instituto Oswaldo Cruz-Fiocruz \\ Av. Brasil 4365 \\ 21045-900 - Rio de Janeiro, RJ \\ ${ }^{2}$ Universidade de Iguaçu - Nova Iguaçu, RJ \\ ${ }^{3}$ UniFoa- Pós-Graduação - Volta Redonda, RJ
}

\begin{abstract}
RESUMO
Foi analisada a ocorrência de babesiose em pequenos roedores nos municípios de Silva Jardim e Nova lguaçu, Estado do Rio de Janeiro. Foram capturados 44 roedores de seis espécies diferentes e entre eles a prevalência da infecção foi de $27,3 \%$. Rattus norvegicus foi considerado o principal reservatório $(50,0 \%)$ e Oligoryzomys nigripes como novo hospedeiro para Babesia sp. Este foi o primeiro relato de Babesia sp. em roedores no Brasil. A freqüência de roedores positivos e o risco de infecção dos roedores não diferiram entre as áreas estudadas.
\end{abstract}

Palavras-chave: Babesia sp., roedores, Brasil

\begin{abstract}
The occurrence of babesiosis was studied in 44 small rodents of six species captured in Silva Jardim and Nova lguaçu counties, State of Rio de Janeiro, Brazil. The prevalence of injection was 27.3\%. Rattus norvegicus was considered as the main reservoir and Oligoryzomys nigripes as a new host to Babesia $s p$. The frequency and the risk of rodent infection were considered equal among the studied areas. This is the first report of Babesia sp in small rodents in Brazil.
\end{abstract}

Keywords: Babesia sp, rodents, Brazil

\section{INTRODUÇÃO}

A babesiose é uma doença cosmopolita e sua transmissão de animais infectados para o homem tem sido bem demonstrada, sendo atualmente considerada uma doença emergente (Brasseur e Gorenflot, 1992), já documentada em muitos países (Montenegro-James, 1992; Gorenflot et al., 1998; Saito-Ito et al., 1999). No Brasil, o primeiro registro de caso de infecção humana por Babesia sp. foi no Estado de Pernambuco, em paciente adulto com quadro clínico de malária benigna (Alecrim et al., 1983). Recentemente, Costa et al. (2000) registraram três novos casos.
A ocorrência de Babesia sp. em roedores pereniza essa zooantroponose em diversas regiões do mundo (Van Peenen et al., 1977; Telford III e Spielman, 1993; Dennis et al., 1998; Fichet-Calvet et al., 2000). No Brasil, até o momento, não há registros desses protozoários em roedores.

A diversidade de nichos ecológicos ocupados dentro de um mesmo habitat, evidencia a capacidade adaptativa dos roedores a diferentes ambientes (Ministério..., 2002). As características ambientais necessárias para o aumento do número de casos em áreas

Recebido para publicação em 13 de maio de 2003

Recebido para publicação, após modificações, em 14 de abril de 2004 gsgazeta@ioc.fiocruz.br 
endêmicas e para a expansão da protozoose para áreas indenes, vêm se formando, principalmente devido ao crescimento desordenado dos centros urbanos, o que resulta na proliferação dos roedores e na maior aproximação entre áreas urbanas e rurais, com difícil delimitação, em determinados momentos, entre essas regiões.

O objetivo deste trabalho foi caracterizar a ocorrência e alguns aspectos epidemiológicos de Babesia sp. em roedores no Brasil, nos municípios de Nova Iguaçu e Silva Jardim, estado do Rio de Janeiro.

\section{MATERIAL E MÉTODOS}

A captura de roedores foi feita a cada 15 dias, entre outubro e novembro de 1999, em ambiente campestre e urbano, nas localidades de Imbaú (S $22^{\circ} 38.372^{\prime}$ WO $42^{\circ} 28.204^{\prime}$ ) e Tinguá (S $22^{\circ} 35.495^{\prime}$ WO $22^{\circ} 24.919^{\prime}$ ), municípios de Silva Jardim e Nova Iguaçu respectivamente. Utilizaram-se 20 armadilhas do tipo francesinha (Carvalho, 1999), distribuídas em transecto em cada área, distando 10 metros uma da outra, no mínimo em dois pontos por esforço de captura, conforme preconizaram Mills et al. (1998). Nas armadilhas, armadas por dois dias consecutivos, foram colocadas iscas de espiga de milho ou mandioca. Os roedores capturados, sedados com algodão embebido em éter sulfúrico comercial, dentro de cuba plástica transparente, foram escovados para a retirada de ectoparasitos conforme recomendação de Carvalho et al. (2001).

Os animais foram acondicionados individualmente em caixas de manutenção apropriadas (Valero, 1990) e encaminhados ao setor de quarentena dos biotérios do Campus III da Universidade Grande Rio e da Universidade Iguaçu, onde permaneceram por 14 dias. Após os primeiros sete dias em cativeiro, foram novamente sedados para obtenção do soro e confecção dos esfregaços sangüíneos. Ao final desse período os animais, silvestres ou campestres, foram liberados em seus locais de origem, desde que apresentassem boas condições de saúde, caso contrário, foram mantidos sob cuidados específicos até seu total restabelecimento. Roedores com hábitos urbanos (Rattus rattus, Rattus norvegicus) foram submetidos ao mesmo procedimento de sedação e coleta de sangue e sacrificados, em seguida, pela inalação de éter sulfúrico.

O sangue foi obtido por punção da artéria ocular, no forame intraorbitário, com auxílio de pipeta Pasteur (Ministério..., 1994). Uma gota do sangue obtido foi utilizada para confecção dos esfregaços, os quais foram encaminhados ao laboratório de Ixodides, Departamento de Entomologia, IOC/Fiocruz - RJ, onde foram fixados em metanol, corados por Giemsa e examinados ao microscópio com objetiva de 100x.

Os testes de Fisher e odds ratio foram utilizados para analisar a positividade entre as regiões estudadas e para avaliar o risco de infecção dos roedores, respectivamente.

A captura, transporte e coleta de material de roedores foi autorizada pelo IBAMA, de acordo com a licença $n^{\circ} 150 / 99$ - DIFAS, Processo $n^{\circ}$ 2001.004961/99-12AC IBAMA.

\section{RESULTADOS E DISCUSSÃO}

Os roedores foram identificados de acordo com a nomenclatura utilizada por Musser e Carleton (1993), conforme os padrões de cariotipagem proposta por Yonenaga et al. (1974). Foram capturados 44 espécimes, pertencentes a seis espécies: Rattus norvegicus Berkenhout, 1769; Nectomys squamipes Brants, 1827, número de cromossomas $(2 \mathrm{n})=56$, número fundamental $(\mathrm{FN})=56$; Oligoryzomys nigripes Wagner, 1845, $2 \mathrm{n}=62, \mathrm{FN}=62 ;$ Oryzomys angoya Hensel, 1872, $2 \mathrm{n}=58, \quad \mathrm{FN}=60 ; \quad$ Holochilus brasiliensis Desmarest, $1817,2 \mathrm{n}=55, \mathrm{FN}=56$; e Akodon cursor Winge, $1888,2 \mathrm{n}=14, \mathrm{FN}=18$.

A prevalência geral de infecção por Babesia sp. foi de $27,3 \%$ ( 12/44), afetando duas espécies, $R$. norvegicus $50 \%(11 / 22)$ e O. nigripes $33,3 \%$ (1/3). Considerando-se a infecção apenas em $R$. norvegicus, não houve diferença entre as localidades avaliadas $(\mathrm{P}=0,59)$, tal como ocorreu ao ser avaliado o risco da mesma espécie nas localidades (odds ratio $=0,27$, intervalo de confiança de 95\%). Quando aplicado o teste odds ratio, considerando a totalidade de exemplares positivos, o risco foi de 0,50 , com intervalo de confiança de 95\%, e variação de 0,1250 a 1,9993 . 
Van Peen et al. (1977) e Shih at al. (1997), em Taiwan, encontraram Rattus coxinga com 74,9\% ( $158 / 211)$ e $66,7 \%$ (8/12) de positivos, respectivamente. A prevalência em Bandicota indica foi de 39,7\% (31/78) (Van Peen et al., 1977). Os resultados encontrados não diferiram muito dos citados na literatura, quanto à prevalência da infecção e freqüência por espécies capturadas. Morsy et al. (1994), na Arábia Saudita, identificaram sete espécies diferentes de roedores com positividade em seis delas: Rattus rattus, 34,5\% (49/142), $R$. norvegicus, 33,3\% (8/24), Mus musculus, 0\% (0/8), Meriones crassus, 33,9\% (19/56), Jaculus jaculus, 31,5\% (5/16), Gerbillus cheesmani, 20\% (10/50) e Acomys c. dimidiatus, $28,6 \%$ (4/14). A prevalência média, $30,6 \%$, é semelhante à encontrada neste trabalho. Krampitz e Baumler (1978), na Alemanha, verificaram prevalência de infecção por Babesia microti de 19\% (99/521) nos roedores capturados. Considerando-se que entre as 11 espécies capturadas apenas uma, Microtus agrestis, apresentou prevalência positiva para infecção por Babesisa microti, $38,9 \%$ (99/255), os resultados deste trabalho e os da Alemanha são próximos. Contudo, quanto às espécies de roedores capturados e às espécies positivas, os resultados deste trabalho são mais elevados. El-Kady et al. (1998), no Egito, observaram 9,1\% (8/88) de infecções por Babesia microti em roedores silvestres, entre cinco espécies capturadas e apenas duas positivas: Acomys c. dimidiatus (6/56) e Dipodillus $d$. dasyurus $(2 / 21)$. A prevalência de captura foi inferior à obtida neste trabalho e a freqüência de espécies positivas, semelhante.

Fichet-Calvet et al. (2000), na Tunísia, encontraram variação estacional na prevalência da infecção por Babesia sp em uma única espécie de roedor - Psammonys obesus. Esses autores citaram prevalência de 26,4\% (101/383), máxima de $48,8 \%$ no mês de agosto. Relataram, ainda, variação inversa entre infecção por Babesia sp. e abundância do roedor. Krampitz e Baumler (1978) também analisaram a sazonalidade da infecção por $B$. microti em roedores na Alemanha. A prevalência máxima ocorreu no início do verão (71\%) e a mínima em janeiro (7\%). Este aspecto não foi avaliado neste estudo pelo curto período de captura.

Todos os trabalhos citados utilizaram o esfregaço sangüíneo corado por Giemsa como método para identificação do parasito. Contudo, Dell'Porto et al. (1990) afirmaram que a infecção por babesia é subestimada quando se utiliza a pesquisa direta e Etkind et al. (1980) relataram que a prevalência encontrada com a observação direta do esfregaço sangüíneo de roedores silvestres pode ser quase dobrada quando se utiliza a inoculação em hamster.

As regiões estudadas apresentam características peculiares por estarem situadas próximas aos grandes centros urbanos e/ou apresentarem grande fluxo populacional. A literatura não apresenta estudos comparativos entre regiões quanto à ocorrência de Babesia sp. em pequenos roedores. $\mathrm{O}$ encontro de roedores infectados por Babesia sp., nas regiões estudadas confirma os estudos de Shih et al.(1997). Esses autores obtiveram positividade para Babesia sp. em roedores que habitam áreas rurais muito próximas às áreas de grande densidade populacional, e chamaram a atenção para o risco do aparecimento de casos de babesiose humana. Esse fato reforça a importância dos achados deste trabalho, já que $R$. norvegicus participa da fauna de roedores urbanos, portanto, convive com o homem graças ao seu comportamento comensal. A emergência de babesiose humana nas Américas (Telford III e Spielman 1993), com o aparecimento de novas espécies de comportamento patogênico mais agressivo, e as evidentes características de maior suscetibilidade para determinados grupos de indivíduos, aumentam a preocupação em identificar os possíveis reservatórios do parasito e seus vetores.

A prevalência apurada para as áreas estudadas é semelhante às de áreas endêmicas e o Rattus norvegicus é o principal reservatório de Babesia $\mathrm{sp}$. entre as espécies de pequenos roedores comensais.

\section{REFERÊNCIAS BIBLIOGRÁFICAS}

ALECRIM, I.; PINTO, B.; ÁVILA, T. et al. Registro do primeiro caso de infecção humana por Babesia spp no Brasil. Rev. Patol. Trop., v.12, p.1129, 1983.

BRASSEUR, P.; GORENFLOT, A. Human babesiosis in Europe. Mem. Inst. Oswaldo Cruz, v.87, p.131-132, 1992.

CARVALHO, R.W. Aspectos ecológicos das faunas de pequenos roedores sinantrópicos e de 
seus sifonápteros ( relação parasito-hospedeiro ) do foco de peste bubônica da serra dos Örgãos de Nova Friburgo, Sumidouro e Teresópolis, Rio de Janeiro, Brasil. 1999. 154f. Tese (Doutorado). Instituto Oswaldo Cruz/FIOCRUZ, Rio de Janeiro, RJ.

CARVALHO, R.W.; SERRA-FREIRE, N.M. et al. Small rodents fleas from the bubonic plague focus located in the Serra dos Órgãos Mountain range, State of Rio de Janeiro, Brazil. Mem. Inst. Oswaldo Cruz, v. 96, p. 603-609, 2001.

COSTA, S.C.; SERRA-FREIRE, N.M.; FRANCO, S. Incidência de babesiose humana no Brasil. In: BIENAL DE PESQUISA DA FIOCRUZ, 2., 2000. Anais... Rio de Janeiro: Fundação Oswaldo Cruz, 2000. P.960 (Resumo).

DELL'PORTO, A.; OLIVEIRA, M.R.; MIGUEL, O. Babesia canis em cães de rua da cidade de São Paulo. I. Estudo comparativo de método de diagnóstico. Braz. J. Vet. Res. Anim. Sci., v.27, p.41-45, 1990.

DENNIS J.W.; TALARICO, J.; CHANG, H.G. et al. Human babesiosis in New York State. Arch. Intern. Med., v.158, p.2149-2154, 1998.

EL-KADY, G.A.; MAKLED, K.M.; MORSY T.A. et al. Rodents, their seasonal activity, ecto-and blood-parasites in Saint Catherine area, South Sinai Govermorate. J. Egypt Soc. Parasitol., v.28, p.81526, 1998.

ETKIND, P.; PIESMAN. J.; RUEBUSH. T.K. et al. Methods for detecting Babesia microti infection in wild rodents. J. Parasitol., v.66, p.107-110, 1980.

FICHET-CALVET, E.; JOMA, A.I.; BEN ISMAIL, R. et al. Patterns of infection of haemoparasites in the fat sand rat, Psammomys obesus, in Tunisia, and effect on the host. Ann. Trop. Med. Parasitol., v.94, p.55-68, 2000.

GORENFLOT, A.; MOUBRI, K.; PRECIGOUT, E. et al. Human babesiosis. Ann. Trop. Med. Parasitol., v.92, p.489-501, 1998.

KRAMPITZ, H.E.; BAUMLER, W. Ocurrence, host range and seasonal prevalence of Babesia microti (Franca, 1912) in rodents of Southern Germany. Z. Parasiten., v.58, p.15-33, 1978.

MILLS J.N.; CHILDS J.E.; KSIAZEK T.G. et al. Método para trampeo y muestreo de pequenos mamíferos para estudios virológicos. In: OPAS REUNIÓN NACIONAL Y EL ENCUENTRO INTERNACIONAL SOBRE SÍNDROME PULMONAR POR HANTAVÍRUS, 2., Washington, DC, 1998. 63p.

MINISTÉRIO da Saúde. Manual de controle da peste. Brasília: Fundação Nacional de Saúde, 1994. $68 \mathrm{p}$.

MINISTÉRIO da Saúde. Manual de controle de roedores. Brasília: Fundação Nacional de Saúde, 2002. 132p.

MONTENEGRO-JAMES, S. Prevalence and control of Babesiosis in the Americas. Mem. Inst. Oswaldo Cruz, v.87, p.27-36, 1992.

MORSY, T.A.; EL BAHRAWY, A.; AL DAKHIL, M.M. et al. Babesia infection in rodents trapped in Riyadh Region, Saudi Arabia, with a general discussion. J. Egypt Soc. Parasitol., v.24, p.177$185,1994$.

MUSSER, G.G.; CARLETON, M.D. Family Muridae. In: WILSON, D.E. Mammal species of the world. A taxonomic and geographic reference. Washington: Smithsonian, 1993. P.501-755.

SAITO-ITO, A.; RAI, S.K.; HE, S.; KOHSAKI, M. et al. First demonstration of Babesia parasitizing in human in Japan. Kansenshogaku Zasshi, v.73, p.1163-1164, 1999.

SHIH, C.M.; LIU, L.P.; CHUNG, W.C. et al. Human babesiosis in Taiwan: asymptomatic infection with a Babesia microti - Like organism in taiwanese woman. J. Clin. Microbiol., v.35, p.450454, 1997.

TELFORD, S.R. III.; SPIELMAN, A. Reservoir competence of white-footed mice for Babesia microti. J. Med. Entomol., v.30, p.223-227, 1993.

VALERO, V.B. (Coord.) Manual para técnicos de biotério. São Paulo: Escola Paulista de Medicina / Finep, 1990. 220p.

VAN PEENEN, P.F.D.; CHANG, S.J.; BANKNIEDER, A.R. et al. Piroplasma from taiwanese rodents. J. Protozool., v.24, p.310-312, 1977.

YONENAGA, Y.; FROTA-PESSOA, O.; KASAHARA, S. et al. Cytogenetic studies on Brazilian rodents. Ciên. Cult., v.28, p.202-211. 1974. 